\section{The Research Associations.}

NOTHING could be more satisfactory than the account that Dr. A. W. Crossley gave on Friday last to the Conference of Research Associations of the constitution and methods of the British Cotton Industry Research Association, of which he is director. It embraces every activity that contributes to the production and utilisation of cotton, and represents more than 95 per cent. of the firms engaged in the industry. Among its members are some of the Labour leaders, and these take the keenest interest in its work. It aims to obtain, in the first place, more exact knowledge of the chemical and physical properties of the fibre and the scientific facts which lie at the base of the processes employed; for it is considered that it is only in this way that the true solution of the problems which present themselves can be assured. It is to be hoped that the same broad and scientific spirit may animate all the associations that have been formed under the Department of Scientific and Industrial Research.

It appeared to be generally agreed that one of the most important conditions of the success of the movement was its close association with the universities and colleges where scientific research has hitherto been mainly carried out. It is to them that research associations and the research departments of private firms must look for their supply of science workers, and it is obviously important that those who are engaged in preparing men and women for the task of industrial research should be acquainted with the lines on which it is carried on. It is for this reason to be desired that the scientific staffs of these institutions should take their share in the technical research required by our industries, and it is a matter of congratulation that the Imperial College of Science and Technology has already led the way in this direction. Lord Crewe, who presided, referred in this connection to the "industrial fellowships" established at Pittsburgh and elsewhere in the United States to facilitate the investigation of technical problems. The work is carried out in close co-operation with the universities, and at the joint expense of the manufacturers concerned and of the endowment.

The question of the publication of the results of industrial research presents serious difficulties. As Dr. Crossley remarked, those employed upon it must keep in close touch with those engaged in pure research, on whose conclusions their work is based, but they cannot be always taking without giving something in return. He urged that a large proportion of the work carried out should ultimately be published even if for commercial reasons it had to be held back for several years; and Dr. Lawrence Balls reminded the conference that the stimulus of the prospect of future publication was required to secure the accurate record of the data obtained in the course of a research.

Not less important are the closely allied questions of the remuneration and superannuation of the scientific workers emploved by the associations. This was discussed by Mr. J. W. Williamson in an interesting paper. $\mathrm{He}$ came to the conclusion that under present conditions $400 l$. per annum is the minimum that should be offered to a science graduate who has already had two or three vears' training in research. He pointed out that a post under a research association did not afford the same security of tenure as one at a university. The desirability of extending to the staffs of research associations the federated superannuation system for universities was acknowledged on all sides.

J. W. E.

\section{Solid Lubricants.}

A LTHOUGH the report of the Lubrication Committee has not yet been issued, a "Memorandum on Solid Lubricants," prepared for the Committee by one of its members, Mr. T. C. Thomsen, has recently been published (Bulletin No. 4 of the Department of Scientific and Industrial Research Advisory Council). This pamphlet of twenty-eight pages contains a digest of the existing knowledge in this branch of the subject, and will be found most useful to all engineers and users of machinery. The solid lubricants referred to are natural and artificial graphite (which are by far the most important), talc, mica, and such sub. stances as flowers of sulphur, white lead, etc., which are occasionally used for curing hot bearings. The greater part of the bulletin is concerned with graphite, and although there is not much matter which is new, there is a great deal of information which will be of interest to many users of lubricants. The action of solid lubricants and the conditions under which they can be usefully employed are clearly explained.

The natural graphite used for lubrication is usually of the flake variety, and varies in the size of its particles from I/Io in. to less than I/200 in. The lubricating graphite produced artificially is amorphous. It is ground even finer than the natural graphite, and by chemical treatment is further reduced to particles of colloidal dimensions and sold under the tradenames of "Aquadag" and "Hydrosol" when in admixture with water, and "Oildag," "Oleosol," and "Kollag" when in admixture with oil. Analyses of the different varieties of lubricating graphites are given in the pamphlet, and it is seen that some are almost chemically pure carbon, whilst others contain mineral matter in variable proportion. Solid lubricants are applied dry in cases where for special reasons it is inadvisable or impossible to use liquid or semisolid lubricants, but they are usually employed in admixture with oil or as an ingredient of greases. When mixed with oil ordinary graphite settles out, owing to its high specific gravity. Colloidal graphite does not settle so long as the vehicle remains neutral, and is carried with oil through the finest orifices, even through worsted trimmings, but it has the disadvantage of being easily caused to coagulate in presence of acid or alkali.

"Oildag" and "Aquadag" have been on the market for a number of years, and the experiences of users of these and other forms of graphite which $\mathrm{Mr}$. Thomsen has collected for general information will be found of considerable value. Perhans the most interesting experience is that of $\mathrm{Mr}$. $\dot{\mathrm{E}}$. W. Johnston, who has successfully emploved "Aquadag" as a cylinder lubricant and eliminated all the trouble caused by the presence of oil in condensed steam. Experiments made at the National Physical Labora. tory showed that the addition of "Oildag " to mineral lubricating oil was advantageous where solid friction occurred, as in worm gear, but quite as good results were obtained with natural flake graphite, so that the lubricating value of graphite seems to depend upon its chemical purity, and the special advantage of the colloidal graphite is due to its pronertv of remaining naturally suspended in the liquid medium without requiring to be stirred constantly bv artificial means. The remarks on the use of graphite in internal. combustion engines, in the lubriration of ropes and chains, and in metal-cutting and wire-drawing will be foind of great interest and nractical use.

All who are interested in lubricants should obtain a copy of this pamphlet, which can be purchased through any bookseller for sixpence. L. A.

$$
\text { No. } 2638 \text {, vOL. I05] }
$$

